\title{
Cherenkov resonances in vortex dissipation in superconductors
}

\author{
B. I. Ivlev and S. Mejía-Rosales \\ Universidad Autónoma de San Luis Potosí, Instituto de Física, A. Obregón 64, 78000 San Luis Potosí, SLP, Mexico \\ M. N. Kunchur \\ Department of Physics and Astronomy, University of South Carolina, Columbia, South Carolina 29208
}

(Received 12 May 1999)

\begin{abstract}
When the velocity of vortices in a superconductor in the free flux flow regime exceeds the velocity of sound, each vortex produces Cherenkov radiation of sound waves. The velocity field of the lattice ions is localized on the Cherenkov cone, but the forces acting on the lattice are localized on the vortex positions. When the Cherenkov cone coincides with certain directions in the moving vortex lattice, the dissipation exhibits maxima. Since the direction of the Cherenkov cone is determined by the vortex velocity (electric field) one can expect a series of resonant peaks in the current-voltage characteristic at particular values of electric field.
\end{abstract}

[S0163-1829(99)02638-7]

\section{INTRODUCTION}

In type-two superconductors the magnetic flux is carried by vortices. If the transport electric current greatly exceeds the pinning critical current $j_{c}$ the current-voltage characteristic ( $I-V$ curve) becomes linear and one might expect such a linearity to hold up to the depairing current $j_{0}$. Nevertheless such a free flux flow regime (FFF) does not extend up to the depairing current. Even with the perfect removal of Joule heat, the finite rate of energy relaxation between the electron system and the lattice leads to a non-equilibrium state. As Larkin and Ovchinnikov ${ }^{1}$ (LO) have shown, such a state, created by a big constant electric field, results in enhancement of superconductivity like under action of microwaves. ${ }^{2}$ The increase of the order parameter is equivalent to a reduction of the vortex core size which, in turn, leads to a deviation of the $I-V$ curve from linearity observed experimentally. ${ }^{3-6}$

The $\mathrm{LO}$ effect is not a unique mechanism for producing a deviation of the $I-V$ curve from linearity. If the vortex velocity exceeds the speed of sound in the crystal the dissipation increases due to Cherenkov emission of sound waves. Each moving vortex creates an electric field acting on the crystal lattice and produces a shock wave (the Cherenkov cone). In a thin film with a magnetic field perpendicular to it the situation is two dimensional and the cone reduces to two lines. The velocity of a crystal lattice is localized on the shock wave but the force acting on the lattice is localized on the vortex positions. The Cherenkov contribution to the total dissipation is proportional to the product of the velocity and the force and hence the dissipation is enhanced when there is a match between vortex positions and the shock wave. If moving vortices are arranged into a vortex lattice then the matching condition can be easily reached when a direction of the shock wave coincides with some direction of the vortex lattice. Since the direction of the shock wave is determined by vortex velocity (electric field) maxima of dissipation can be reached at some particular values of the electric field. According to this, the $I$ - $V$ curve should have a series of maxima as a function of voltage. This is the origin of the
Cherenkov resonances under vortex motion.

A necessity for sharp resonances is the existence of a relatively perfect lattice of moving vortices. At high vortex velocities this is indeed the case, through the well-known process of dynamic crystallization. ${ }^{7-9}$ Thus in the regime of highly driven flux flow the resonances ought to be observable.

\section{CRYSTAL LATTICE DYNAMICS}

Consider a three-dimensional superconductor in which $B$ is directed along the $z$ axis. At sufficiently high transport current density $j$ there is a free flow of vortices with the velocity

$$
\mathrm{v}=c \frac{E}{B} .
$$

If $v$ exceeds the speed of sound one can expect the Cherenkov effect resulting in some peculiarities in the currentvoltage characteristics. The equation of motion for a lattice displacement $\vec{u}$ has the form

$$
\frac{\partial^{2} \vec{u}}{\partial t^{2}}+\gamma \frac{\partial \vec{u}}{\partial t}-s^{2}\left(\frac{\partial^{2} \vec{u}}{\partial x^{2}}+\frac{\partial^{2} \vec{u}}{\partial y^{2}}\right)=\frac{1}{\rho_{0}} \vec{f}(\vec{r}-\overrightarrow{\mathrm{v}} t),
$$

where $\vec{f}$ is a force density acting on a crystal lattice from electrons and $\rho_{0}$ is a crystal density. $\vec{f}$ will be specified later. $\vec{u}$ is the longitudinal displacement and $s$ is the velocity of longitudinal sound. (As we will show below, the effect of the longitudinal part of $\vec{f}(\vec{r})$ is more important than the transverse one.)

The sound attenuation is represented phenomenologically in Eq. (2) through the coefficient $\gamma$. In the Fourier representation $\gamma=\alpha s k$, where $\alpha \simeq s / \mathrm{v}_{F} \cdot{ }^{10}$

With the new variable $\vec{\rho}=\vec{r}-\vec{v} t$ one can find a solution of Eq. (2) taking $\overrightarrow{\mathrm{v}}$ to be along $x$ and $\mathrm{v}>s$ : 


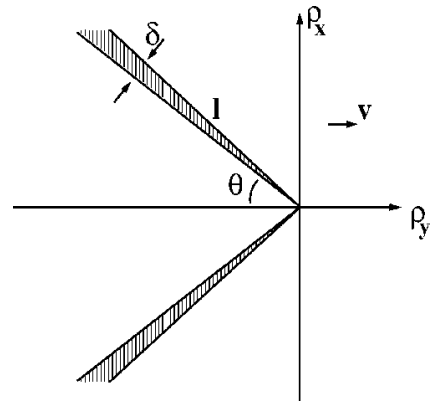

FIG. 1. Schematic representation of the Cherenkov cone. The width $\delta$ is given in the text. The angle $\theta$ is determined by the condition $\sin \theta=s / \mathrm{V}$.

$$
\vec{u}(\vec{\rho})=-\frac{1}{2 \rho_{0} s^{2} \lambda} \int d^{2} R \vec{f}(\vec{\rho}+\vec{R}) \Phi(\vec{R}),
$$

where

$$
\Phi(\vec{R})=\int_{-\infty}^{\infty} \frac{d k_{y}}{\pi k_{y}} \exp \left(-i k_{y} R_{y}-\frac{\beta}{\lambda}\left|k_{y}\right| R_{x}\right) \Theta\left(R_{x}\right)
$$

and $\lambda=\left(\mathrm{V}^{2} / s^{2}-1\right)^{1 / 2}$ and $2 \beta=\alpha \mathrm{V}^{2} /\left(\mathrm{V}^{2}-s^{2}\right)$.

If $f(\vec{\rho}) \sim \delta(\vec{\rho})$ the solution (3) corresponds to a Cherenkov cone propagating in the $x$ direction with the velocity $\mathrm{v}$. In the limit of small attenuation $\beta \rightarrow 0, \quad \vec{u}(\vec{\rho})$ $\simeq \Theta\left[\left|\rho_{y}\right|\left(\mathrm{V}^{2} / s^{2}-1\right)^{1 / 2}-\rho_{x}\right]$. The Cherenkov cone produced by a single vortex is shown in Fig. 1. The crystal lattice displacement $u=0$ to the right of the cone and to the left is

$$
u \sim \frac{\hbar}{p_{F}} \frac{s}{\mathrm{v}_{F}} \frac{\mathrm{v}}{\sqrt{\mathrm{v}^{2}-s^{2}}}
$$

Of course, far to the left of the cone there is the boundary condition $u=0$ since the crystal does not move as a whole. This boundary condition does not affect the solution in the vicinity of the cone. The width of the shock wave due to sound attenuation is

$$
\delta \sim \frac{\alpha s}{\sqrt{\mathrm{v}^{2}-s^{2}}} l,
$$

where $l$ is the length along the cone.

The additional dissipation density due to shock wave motion is

$$
w=\int \frac{d^{2} \rho}{S} \vec{f} \frac{\partial \vec{u}}{\partial t},
$$

where $S$ is the surface area of the sample. Since $\partial \vec{u} / \partial t$ $=-\mathrm{v} \partial \vec{u} / \partial \rho_{x}$, it follows from Eqs. (7) and (3)

$$
w=-\frac{\mathrm{v}}{2 \rho_{0} s^{2} \lambda} \int d^{2} R \Phi(\vec{R}) \int \frac{d^{2} \rho}{S} \vec{f}(\vec{\rho}) \frac{\partial \vec{f}(\vec{\rho}+\vec{R})}{\partial R_{x}} .
$$

The total force density is

$$
\vec{f}(\vec{\rho})=\sum_{i} \vec{f}^{(0)}\left(\vec{\rho}-\vec{r}_{i}\right)
$$

where $\vec{r}_{i}$ are coordinates of vortices and $\vec{f}^{(0)}$ corresponds to the force from a single vortex. According to this, Eq. (8) has the form

$$
\begin{aligned}
w= & \frac{\mathrm{v}}{2 \rho_{0} s^{2} \lambda} \int \frac{d^{2} R}{S} \Phi(\vec{R}) \\
& \times \int \frac{d^{2} k}{(2 \pi)^{2}} i k_{x} e^{-i \vec{k} \vec{R}} \vec{f}_{k}^{(0)} \vec{f}_{-k}^{(0)} \sum_{i, j} e^{i \vec{k}\left(\vec{r}_{i}-\vec{r}_{j}\right)} .
\end{aligned}
$$

If moving vortices are organized into a vortex lattice then

$$
w=\frac{\mathrm{v}}{2 \rho_{0} s^{2} \lambda}\left(\frac{B}{\Phi_{0}}\right)^{2} \sum_{b} i b_{x} \vec{f}_{b}^{(0)} \vec{f}_{-b}^{(0)} \int d^{2} R \Phi(\vec{R}) e^{-i \vec{b} \vec{R}},
$$

where $\vec{b}$ is a reciprocal vector of the vortex lattice and $\Phi_{0}$ $=\pi \hbar c / e$ is the flux quantum.

Making the $R$ integration in Eq. (11), one can obtain

$$
w=\frac{1}{\rho_{0} S}\left(\frac{B}{\Phi_{0}}\right)^{2} \sum_{b_{x} \neq 0} \vec{f}_{b}^{(0)} \vec{f}_{-b}^{(0)} \frac{i b_{x}}{\left(\lambda b_{x}+i \beta\left|b_{y}\right|\right)^{2}-b_{y}^{2}} .
$$

\section{FORCE ON A CRYSTAL LATTICE}

The force acting on a crystal lattice by the electronic system can be written as

$$
\vec{f}^{(0)}=e n \vec{E},
$$

where $n$ is the crystal density and $\vec{E}$ is the electric field produced by vortex motion. ${ }^{11-13} \mathrm{We}$ ignore the Lorentz force since it does not contribute to dissipation and we omit the drag force on the crystal lattice due to impurities. We also neglect the drag force on vortices due to pinning ${ }^{14}$ since we are concerned with vortex motion in the free flux flow limit.

The longitudinal part of $\vec{E}\left(\nabla \times \vec{E}_{\text {longitudinal }}=0\right)$ is localized within a distance much less than the London penetration depth. In this region the electric field has the form ${ }^{15}$

$$
\vec{E}=\frac{\hbar}{2 e} \nabla \dot{\chi}-\nabla \Phi, \quad \nabla \chi(\vec{\rho})=\frac{1}{\rho^{2}}\left(\begin{array}{c}
-\rho_{y} \\
\rho_{x}
\end{array}\right),
$$

where $\chi$ is the singular vortex phase and $\Phi$ is the gauge invariant scalar potential. $\Phi(\vec{\rho})$ is screened on the distance $l_{E}$ which is the penetration depth of the longitudinal electric field in superconductors. For simplicity we take the BardeenStephen approach and put $l_{E} \sim \xi$, where $\xi$ is the coherence length. According to this, on the distance bigger than $\xi$ from the vortex core one can use the expression $\vec{E}=(\hbar / 2 e) \nabla \dot{\chi}$ and since $\dot{\chi}=-\mathrm{v} \partial \chi / \partial \rho_{x}$ in the Fourier components,

$$
\vec{E}_{k}=\frac{\mathrm{v} \Phi_{0}}{c} \frac{k_{x}}{k^{2}}\left(\begin{array}{c}
k_{y} \\
-k_{x}
\end{array}\right), k \ll \frac{1}{\xi} .
$$

\section{THE CHERENKOV DISSIPATION}

Substitution of Eq. (15) into Eq. (12) yields 


$$
\begin{aligned}
w= & \frac{\mathrm{v}^{3}}{4 s^{3} \lambda}\left(\frac{B}{H_{c 2}}\right)^{2} n\left(\frac{n}{\rho_{0}} \frac{\hbar^{2}}{\xi^{2}}\right) \frac{s}{\xi^{2}} \\
& \times \sum_{b_{x} \neq 0} \frac{1}{b^{2}} \frac{i b_{x}^{3}}{\left(\lambda b_{x}+i \beta\left|b_{y}\right|\right)^{2}-b_{y}^{2}}\left(\frac{1}{1+b^{2} \xi^{2} / 2 \pi}\right),
\end{aligned}
$$

where $H_{c 2}=\Phi_{0} / 2 \pi \xi^{2}$ is the upper critical field. The last bracket term in Eq. (16) is introduced according to cutoff at $k \sim 1 / \xi$.

To calculate the $b$ sum in Eq. (16) let us suppose that moving vortices are arranged into a square lattice $\left\{b_{x}, b_{y}\right\}$ $=2 \pi\{m, n\} \sqrt{B / \Phi_{0}}$. Then the $b$ sum in Eq. (16) has the form

$$
\begin{aligned}
& \xi \sqrt{\frac{H_{c 2}}{2 \pi B}} \frac{s^{2} \lambda}{\mathrm{v}^{2}} \sum_{m \neq 0} \frac{i m}{1+\left(\mathrm{v}^{2} / s^{2}\right)\left(B / H_{c 2}\right) m^{2}} \\
& \times \sum_{n \neq 0} \frac{1}{\lambda^{2}(m+i \beta|m|)^{2}-n^{2}} .
\end{aligned}
$$

The $n$ summation can be done easily. Considering $\alpha$ to be a small parameter one can write by means of Eq. (16) and expression (17)

$$
w=\frac{s}{\alpha \mathrm{v} \sqrt{2 \pi}}\left(\frac{B}{H_{c 2}}\right)^{3 / 2} n\left(\frac{n}{\rho_{0}} \frac{\hbar}{\xi^{2}}\right) \frac{s}{\xi} Y\left(\frac{\mathrm{v}}{s}\right),
$$

where

$$
\begin{aligned}
Y(x)= & \frac{1}{x^{2}} \sum_{n=1}^{\infty} \frac{1}{n}\left(\frac{1}{1+n^{2} x^{2} B / H_{c 2}}\right) \\
& \times \frac{1}{1+(2 / \pi \alpha)^{2}\left[\tan \left(\pi n \sqrt{x^{2}-1}\right)\right]^{2}\left(x^{2}-1\right) / n^{2} x^{4}} .
\end{aligned}
$$

Equation (19) is valid under the condition $x^{2}-1 \gg \alpha^{2} n^{2} x^{4}$. For $x \geq 1$, typical $n$ is of the order $\sqrt{H_{c 2} / B}$ and for $x \gg 1$ one should take $n=1$. According to this, the applicability condition of Eqs. (18) and (19) is

$$
\frac{1}{\alpha^{2}} \frac{H_{c 2}}{B}<\left(\frac{\mathrm{v}}{s}-1\right) ; \quad \frac{\mathrm{v}}{s} \ll \frac{1}{\alpha} .
$$

Equations (16) and (18) relate to the case $\mathbf{V}>s$. In the opposite case $\mathrm{V}<s$ one can easily show that instead of Eq. (16) we have

$$
\begin{aligned}
w= & \frac{\alpha}{4}\left(\frac{\mathrm{v}}{s}\right)^{5}\left(\frac{B}{H_{c 2}}\right)^{2} n\left(\frac{n}{\rho_{0}} \frac{\hbar^{2}}{\xi^{2}}\right) \frac{s}{\xi^{2}} \\
& \times \sum_{b_{x} \neq 0} \frac{\left|b_{x}\right| b_{x}^{4}}{b^{2}\left[b_{y}^{2}+\left(1-\mathrm{v}^{2} / s^{2}\right) b_{x}^{2}\right]^{2}} \frac{1}{1+b^{2} \xi^{2} / 2 \pi} .
\end{aligned}
$$

The main contribution at $\left(1-\mathrm{v}^{2} / s^{2}\right) \ll 1$ comes from terms with $b_{y}=0$. The result is

$$
\begin{aligned}
w= & \frac{\alpha}{4 \sqrt{2 \pi}}\left(\frac{B}{H_{c 2}}\right)^{3 / 2}\left(\ln \frac{H_{c 2}}{B}\right) \frac{s^{4}}{\left(s^{2}-\mathrm{v}^{2}\right)^{2}} n\left(\frac{n}{\rho_{0}} \frac{\hbar^{2}}{\xi^{2}}\right) \frac{s}{\xi} \\
& {[0<(1-\mathrm{v} / s) \ll 1] . }
\end{aligned}
$$

Equations (18) and (22) give the Cherenkov dissipation for moving vortices arranged in a lattice.

\section{CURRENT-VOLTAGE CHARACTERISTIC}

The total dissipation $\vec{j} \vec{E}$ consists of two parts:

$$
\vec{j} \vec{E}=\frac{H_{c 2}}{B} \frac{\vec{E}^{2}}{\rho}+w .
$$

The first term in the right-hand side of Eq. (23) is the conventional dissipation under vortex motion which occurs mainly close to the vortex core. We have used the BardeenStephen approximation for this dissipation. The second term in the right-hand side of Eq. (23) is the Cherenkov dissipation calculated above. We omit here the distortion of the $I-V$ curve by the LO effect since away from LO instability the non-Cherenkov part of current-voltage characteristic is linear. ${ }^{1}$ Since the vortex velocity obeys Eq. (1), where $E$ is the mean electric field, the current-voltage characteristics has the form

$j=\frac{H_{c 2}}{B} \frac{E}{\rho}+\sqrt{\frac{2 B}{\pi H_{c 2}}} e n\left(\frac{\hbar}{M \xi}\right)$

$$
\times \begin{cases}\frac{\alpha}{16\left(1-E / E_{0}\right)^{2}} \ln \frac{H_{c 2}}{B} ; & 0<\left(E_{0}-E\right) \ll E_{0} \\ \frac{1}{\alpha} Y\left(\frac{E}{E_{0}}\right) ; & E_{0}<E .\end{cases}
$$

Here $E_{0}=B s / c$ and $M=\rho_{0} / n$ is the average ion mass. One can use the following estimates $M \simeq m\left(\mathrm{~V}_{F} / s\right)^{2}$, where $m$ is the electron mass, $\xi \simeq 15 \AA, \rho \simeq 10^{-4} \Omega \mathrm{cm}$, and $\mathrm{v}_{F} / s$ $\simeq 10^{2}$. This gives a current-voltage characteristic close to $E_{0}$ of the form

$10^{-7} j\left(\mathrm{~A} / \mathrm{cm}^{2}\right)$

$$
\begin{aligned}
=1 & +10^{-21} \sqrt{\frac{B}{H_{c 2}}} n\left(\mathrm{~cm}^{-3}\right) \\
& \times \begin{cases}10^{-5}\left(\frac{E_{0}}{E_{0}-E}\right)^{2} \ln \frac{H_{c 2}}{B} ; & 0<\left(E_{0}-E\right) \ll E_{0} \\
Y\left(\frac{E}{E_{0}}\right) ; & E_{0}<E .\end{cases}
\end{aligned}
$$

The plot of the function $Y\left(E / E_{0}\right)$ at $B / H_{c 2}=0.1$ and $\alpha$ $=10^{-2}$ is shown in Fig. 2. The main peak is positioned at $E / E_{0}=\sqrt{2}$ and has a width $\delta E / E_{0} \sim \alpha$ like some other peaks in the region $E / E_{0} \sim 1$. This is valid for sufficiently perfect moving lattice of vortices. If $\vec{R}$ is the equilibrium vortex position in the perfect lattice the deviation from it due to disorder $\delta R(\vec{R})$ should satisfy the condition $\delta R(\vec{R}) / R \leqslant \alpha$ at $R \sim \sqrt{\Phi_{0} / B}$. For bigger deviations $\delta R$ the peaks in Fig. 2 are more smeared and for a completely uncorrelated vortex system the curve becomes smooth as is shown in Sec. VI. 


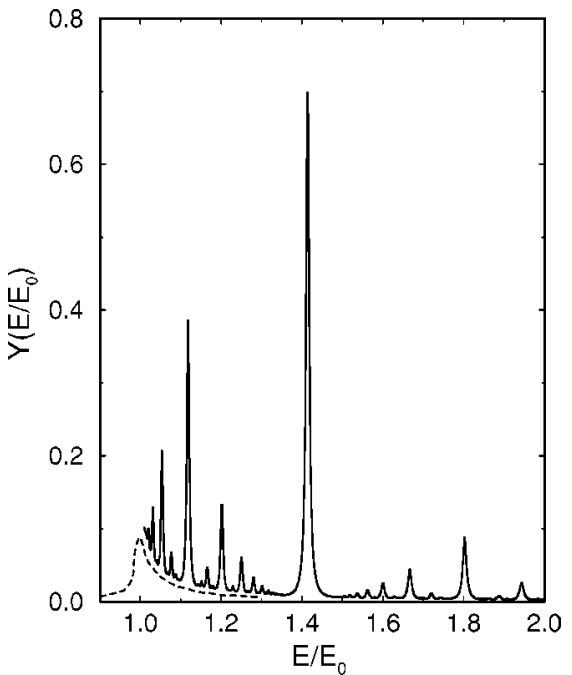

FIG. 2. The plot of the function $Y\left(E / E_{0}\right)$ which determines, according to Eqs. (24) and (25), the $I-V$ characteristic in the case of the moving vortex lattice. Parameters are chosen as $B / H_{c 2}=0.1$ and $\alpha=10^{-2}$. The dashed line, corresponding to the unphysical case of an uncorrelated moving vortex gas, is plotted just to show the limiting positions of current-voltage characteristics.

\section{UNCORRELATED VORTEX SYSTEM}

In the previous section we have considered the motion of vortices arranged in a perfect lattice, leading to the resonant behavior shown in Fig. 2. However, a violation of the translational invariance of the vortex lattice leads to a smearing of the peaks. The physical origin of peaks is the inclusion of a large number of other vortices within the Cherenkov cone width. In reality a moving vortex system will not be a perfect lattice, due to the effects of thermal and quenched (pinning) disorders. Rather than analyzing the smearing of peaks in a quantitative manner, we consider instead the opposite limiting case of a completely uncorrelated (amorphous/liquid) vortex system. Although this limiting case is not physical, ${ }^{7-9}$ it nevertheless establishes a useful limit. The real situation, and the corresponding current-voltage curve, will lie between the two limiting cases of perfect lattice and uncorrelated vortices.

In the case of uncorrelated vortices the average of Eq. (10) reads

$$
\left\langle\sum_{i, j} e^{i \vec{k}\left(\vec{r}_{i}-\vec{r}_{j}\right)}\right\rangle=\frac{B}{\Phi_{0}} S
$$

Equations (16) and (21) become modified by the substitution $\Sigma \rightarrow \int d^{2} k /(2 \pi)^{2}$. The $\vec{k}$ integration is simple and the result for uncorrelated vortices is

$$
j=\frac{H_{c 2}}{B} \frac{E}{\rho}+\pi e n\left(\frac{\hbar}{M \xi}\right) \begin{cases}\frac{\alpha}{\pi} g\left(\frac{E}{E_{0}}\right) ; & E<E_{0} \\ \frac{E_{0}}{E \sqrt{E^{2}-E_{0}^{2}}} ; & E_{0}<E,\end{cases}
$$

where

$$
\begin{aligned}
g(x) & =3+\frac{x^{2}}{1-x^{2}}-\frac{3-4 x^{2}}{x\left(1-x^{2}\right)^{3 / 2}} \arcsin x \\
& =\left\{\begin{array}{cc}
\frac{\pi}{2} \frac{1}{\left(1-x^{2}\right)^{3 / 2}} & (1-x) \ll 1 \\
\frac{16 x^{4}}{15} & x \ll 1 .
\end{array}\right.
\end{aligned}
$$

Close to $E_{0}$ the current-voltage characteristic can be presented as the following:

$$
\begin{aligned}
10^{-7} j\left(\mathrm{~A} / \mathrm{cm}^{2}\right)=1 & +10^{-23} n\left(\mathrm{~cm}^{-3}\right) \\
& \times\left\{\begin{array}{cl}
\frac{\alpha}{4\left(1-E / E_{0}\right)^{3 / 2}} ; & E<E_{0} \\
\frac{1}{\sqrt{E / E_{0}-1}} ; & E_{0}<E .
\end{array}\right.
\end{aligned}
$$

The crossover between two cases occurs at $\left|E / E_{0}-1\right|$ $\sim \alpha / 4 \sim 10$. Here there is only one peak at $E=E_{0}$. At $B / H_{c 2}=0.1$ the curve for uncorrelated vortices is represented by the dashed line in Fig. 2.

\section{CONCLUSIONS}

The Cherenkov effect can be observed at sufficiently high voltage across a sample. Equation (1) with $\mathrm{V} \simeq s$ gives an estimate $E \simeq B s / c$ for observation of the Cherenkov effect. For a typical sound velocity in metals $s \simeq 10^{5} \mathrm{~cm} / \mathrm{s}$ the necesary electric field is $E(\mathrm{~V} / \mathrm{cm}) \simeq 10 B(\mathrm{~T})$. In a current-biased measurement, the series of resonances become replaced by a single step in dissipation at $\mathbf{V} \simeq s$. Preliminary evidence of this has been observed recently in connection with the study of low-temperature flux flow in $\mathrm{YBa}_{2} \mathrm{CuO}_{7}$ films. ${ }^{16}$

\section{AKNOWLEDGMENTS}

We are grateful to D. Christen for valuable discussions. M.N.K. acknowledges support from the U.S. Department of Energy through Grant No. DE-FG02-99ER45763.
${ }^{1}$ A.I. Larkin and Yu.N. Ovchinnikov, Zh. Éksp. Teor. Fiz. 68, 1915 (1975) [Sov. Phys. JETP 41, 960 (1976)].

${ }^{2}$ B.I. Ivlev, S.G. Lisitsyn, and G.M. Eliashberg, J. Low Temp. Phys. 10, 449 (1973).

${ }^{3}$ L.E. Musienko, I.M. Dmitrenko, and V.G. Volotskaya, Pis'ma Zh. Éksp. Teor. Fiz. 31, 603 (1980) [JETP Lett. 31, 567 (1980)].
${ }^{4}$ W. Klein, R.P. Huebener, and J. Parisi, J. Low Temp. Phys. 61, 413 (1985).

${ }^{5}$ S.G. Doettinger, R.P. Huebener, R. Gerdemann, A. Kuhle, S. Anders, T.G. Trauble, and J.C. Villegier, Phys. Rev. Lett. 73, 1691 (1994).

${ }^{6}$ M.N. Kunchur, B.I. Ivlev, D.K. Christen, and J.M. Phillips (unpublished). 
${ }^{7}$ P. Thorel, R. Kahn, Y. Simon, and D. Cribier, J. Phys. (Paris) 34, 447 (1973).

${ }^{8}$ U. Yaron, P.L. Gammel, D.A. Huse, R.N. Kleiman, C.S. Oglesby, E. Bucher, B. Battlogg, D.J. Bishop, K. Mortensen, K. Clausen, C.A. Bolle, and F. De La Cruz, Phys. Rev. Lett. 73, 2748 (1994).

${ }^{9}$ A. Duarte, E. Fernandez Righi, C. A. Bolle, F. de la Cruz, P.L. Gammel, C.S. Oglesby, B. Bucher, B. Batlogg, and D.J. Bishop, Phys. Rev. B 53, 11336 (1996).

${ }^{10}$ A.A. Abrikosov, Fundamentals of the Theory of Metals (NorthHolland, Amsterdam, 1988).

${ }^{11}$ V.M. Kontorovich, Zh. Éksp. Teor. Fiz. 45, 1638 (1963) [Sov.
Phys. JETP 18, 1125 (1964)].

${ }^{12}$ D.P. Belozorov and E.A. Kaner, Zh. Éksp. Teor. Fiz. 55, 642 (1968) [Sov. Phys. JETP 28, 334 (1969)].

${ }^{13}$ I.E. Bulyzhenkov and B.I. Ivlev, Zh. Éksp. Teor. Fiz. 71, 1172 (1976) [Sov. Phys. JETP 44, 613 (1976)].

${ }^{14}$ J. Pankert, G. Marbach, A. Comberg, P. Lemmens, P. Froning, and S. Ewert, Phys. Rev. Lett. 65, 3052 (1990).

${ }^{15}$ L.P. Gorkov and N.B. Kopnin, Zh. Éksp. Teor. Fiz. 65, 396 (1973) [Sov. Phys. JETP 38, 195 (1974)].

${ }^{16}$ M.N. Kunchur, B.I. Ivlev, D.K. Christen, and J.M. Phillips, Bull. Am. Phys. Soc. 44, 1703 (1999). 